\title{
Bilateral Retinal Detachment after Implantable Collamer Lens Surgery
}

\author{
Mohamad Rosman* and Tong Weihan \\ Singapore National Eye Centre, Singapore
}

Received: 眥 April 04, 2018; Published: 制 April 19, 2018

*Corresponding author: Mohamad Rosman, Singapore National Eye Centre, Refractive Surgery (Head of Department), Singapore

\begin{abstract}
A 46-year-old man, with moderate myopia, underwent Implantable Collamer Lens (ICL) surgery in both eyes on different dates. In the post-operative period, both of his eyes sustained the complication of rhegmatogenous retinal detachment (RD). After RD in the first eye, prophylactic 360-degree laser photocoagulation was performed on the second eye pre-operatively to hopefully reduce the risk of RD. This did not prevent RD from developing in this eye as well. RD is a potential complication of ICL surgery and all patients, regardless of degree of myopia, should be counselled about the risk pre-operatively. It will be prudent to monitor patients closely in the post-operative period, to detect this potential complication early. With subsequent early intervention, patients can have a good final visual outcome.
\end{abstract}

Keywords: Implantable Collamer Lens; Retinal detachment; Myopia; Laser photocoagulation

Abbreviations: ICL: Implantable Collamer Lens; RD: Retinal Detachment; PIOL: Phakic Intraocular Lenses; LASIK: Laser Assisted in Situ Keratomileusis; VA: Visual Acuity

\section{Introduction}

Various modern surgical options exist for correcting patients' refractive errors. They range from minimally invasive surgery, such as Laser-assisted In Situ Keratomileusis (LASIK), to more invasive options such as Refractive Lens Exchange. To date, LASIK remains one of the most well-known and commonly performed procedures globally. Phakic intraocular lenses (PIOL), one of the more commonly used types being the Implantable Collamer Lens (ICL), remain a viable option for treating higher refractive error than that which can be safely managed by corneal refractive surgery. PIOL implantation also has the advantage of preserving natural accommodation. Its removable nature makes its refractive effect theoretically reversible. Retinal detachment (RD) is a known potential complication of ICL surgery, though relatively uncommon. We present such a case in the following section.

\section{Case Description}

A 46-year-old male with moderate myopia as his only ocular history presented to the refractive surgery service. He was keen for refractive surgery to avoid using glasses. His initial best corrected distance visual acuity (VA) was 6/6 bilaterally. Manifest refraction revealed -6.00D spherical and-1.00D cylindrical components in the right eye, and $-5.00 \mathrm{D}$ spherical and $-1.50 \mathrm{D}$ cylindrical components in his left eye. Intraocular pressures were normal, the corneas were healthy, and the anterior chambers were deep and quiet. The lenses were clear. Examination of the posterior segment was unremarkable. The optic nerves were healthy as well. Refractive surgical options were discussed. He opted for ICL surgery over LASIK, to preserve the option of multifocal intraocular lenses for future cataract surgery. Risks of surgery were advised, including anaesthesia complications, possibilities of blindness, retinal detachment, endophthalmitis, corneal decompensation, early cataract formation and refractive surprise. Pre-operative investigations, including endothelial cell count and ultrasound biomicroscopy, were normal. Bilateral laser peripheral iridotomies were done pre-operatively to prevent pupillary block. The patient subsequently underwent ICL implantation in his right eye. No issues were noted on post-operative day 1 .

On post-operative day 4 , the patient noticed a new onset of right eye floaters and blurring of vision. On examination, a right eye macula-sparing rhegmatogenous RD was diagnosed. He then 
underwent vitrectomy with pneumatic retinopexy for RD repair. Retinal reattachment ensued uneventfully. Fortunately, best corrected VA recovered to 6/7.5.

Due to anisometropia after unilateral ICL implantation, the patient was keen for ICL implantation in the left eye soon after right eye recovery. In view of his recent right-sided post-operative RD, he was counselled extensively regarding the risk of RD occurrence in the left eye. He underwent 360-degree laser photocoagulation intended to hopefully reduce the risk of RD recurrence, followed by ICL surgery for the left eye. One month after left eye ICL insertion, he presented with acute left eye blurring of vision. Examination revealed a macula-sparing superior rhegmatogenous RD (Figures $1,2 \& 3)$. He subsequently required two retinal detachment repair operations to achieve retinal reattachment for his left eye.



Figure 1: Superior rhegmatogenous retinal detachment of left eye.

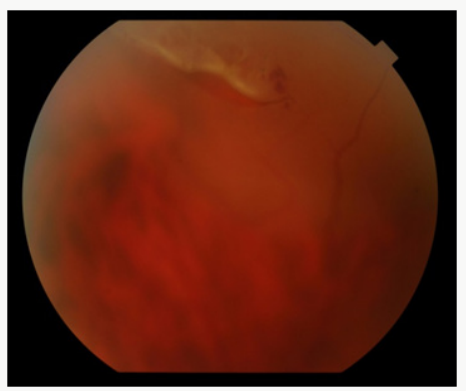

Figure 2: Superior retinal tear of left eye.



Figure 3: Posterior fundus of left eye with no macular involvement.

Both eyes recovered well. 6 months after his second RD, manifest refraction showed best corrected VA of 6/7.5 bilaterally.
He later developed left eye posterior subcapsular cataract, and underwent uneventful phacoemulsification with ICL removal 1 year after his RDs. His final VA was $6 / 6$ bilaterally after left eye cataract surgery. Final manifest refraction showed $-0.75 \mathrm{D}$ spherical and -0.50 cylindrical components for the right eye, and $+0.25 \mathrm{D}$ spherical and $-0.25 \mathrm{D}$ cylindrical components for the left eye.

\section{Discussion}

ICL implantation has many known potential complications, including but not limited to cataract formation, pigmentary dispersion, and endothelial cell loss. Cataract formation remains the most frequent complication [1]. RD is relatively uncommon but not unheard of. Fortunately in most cases, RD repair surgery can be carried out safely while preserving the PIOL [2]. The risk of post-operative RD is generally low across various studies of PIOL implantation [3-6]. One study found the incidence to be $1.5 \%$ in 530 eyes over a two-year follow-up period [6]. Surgical reattachment was achieved in the eyes affected by RD, with mean best corrected VA of 6/24 after RD repair [6]. Authors have previously described the occurrence of RD in ICL implantation, but only in those with high myopia, such as a case in a patient's eyes with $-15.00 \mathrm{D}$ and -21.00D [7]. This is due to RD having a tendency to occur in eyes with greater axial length [5]. The patient in our case only had moderate myopia, but RD still occurred. This may suggest that the risk exists in all myopic patients, and not only in those with high myopia. Hence, it is essential that all patients are advised regarding this risk.

In our case, the patient developed a RD in his right eye as soon as 4 days after ICL implantation. The earliest onset of RD ever described after ICL insertion was 3 hours [8]. Given the possibility of rapid RD onset, it would be prudent to monitor patients closely in the post-operative period. This will allow a RD, should it occur, to be treated sooner rather than later. After the patient suffered his first RD, even with prophylactic 360-degree laser done for his second eye, he still developed a post-operative RD in this eye. Preventive measures seek to hopefully reduce the risk of RD, but it is possible that in certain patients, the risk of RD remains high despite prior prophylactic laser photocoagulation. This case report highlights the potential risk of RD in ICL surgery, and the need to always counsel patients regarding this risk. This holds true not only for highly myopic patients, but for all myopic patients. RD can occur very early post-operatively, hence it is vital to warn patients regarding RD symptoms and to monitor patients closely. If detected early, treatment can be instituted quickly, which can lead to good visual outcomes.

\section{References}

1. Fernandes P, Gonzalez Meijome JM, Madrid Costa D (2011) Implantable collamer posterior chamber intraocular lenses: a review of potential complications. Journal of refractive surgery 27(10): 765-776.

2. Kim YJ, Chung JK, Lee SJ (2014) Retinal detachment surgery in eyes with iris-fixated phakic intraocular lenses: Short-term clinical results. Journal of cataract and refractive surgery 40(12): 2025-2030.

3. Al Abdullah AA, Al Falah MA, Al Rasheed SA (2015) Retinal Complications After Anterior Versus Posterior Chamber Phakic Intraocular Lens 
Implantation in a Myopic Cohort. Journal of refractive surgery 31(12): 814-819.

4. Bamashmus MA, Al Salahim SA, Tarish NA (2013) Posterior vitreous detachment and retinal detachment after implantation of the Visian phakic implantable collamer lens. Middle East African journal of ophthalmology 20(4): 327-331.

5. Ruiz Moreno JM, Montero JA, De la Vega C (2006) Retinal detachment in myopic eyes after phakic intraocular lens implantation. Journal of refractive surgery 22(3): 247-252.
6. Jiang T, Chang Q Wang X (2012) Retinal detachment after phakic intraocular lens implantation in severe myopic eyes. Graefe's archive for clinical and experimental ophthalmology, Albrecht von Graefes Archiv fur klinische und experimentelle Ophthalmologie 250(12): 1725-1730.

7. Mushawiahti M, Shaw A, Chiu D (2014) Retinal detachment associated with posterior chamber phakic intraocular lens implantation. Clinical \& experimental optometry 97(5): 471-472.

8. Domenech NP, Arias L, Prades S, Octavi Pujol, Marc Rubio, et al. (2008) Acute onset of retinal detachment after posterior chamber phakic intraocular lens implantation. Clinical ophthalmology 2(1): 227-231.

\section{(c) \\ This work is licensed under Creative Commons Attribution 4.0 License}

To Submit Your Article Click Here:

Submit Article

DOI: $10.32474 /$ TOOAJ.2018.01.000110

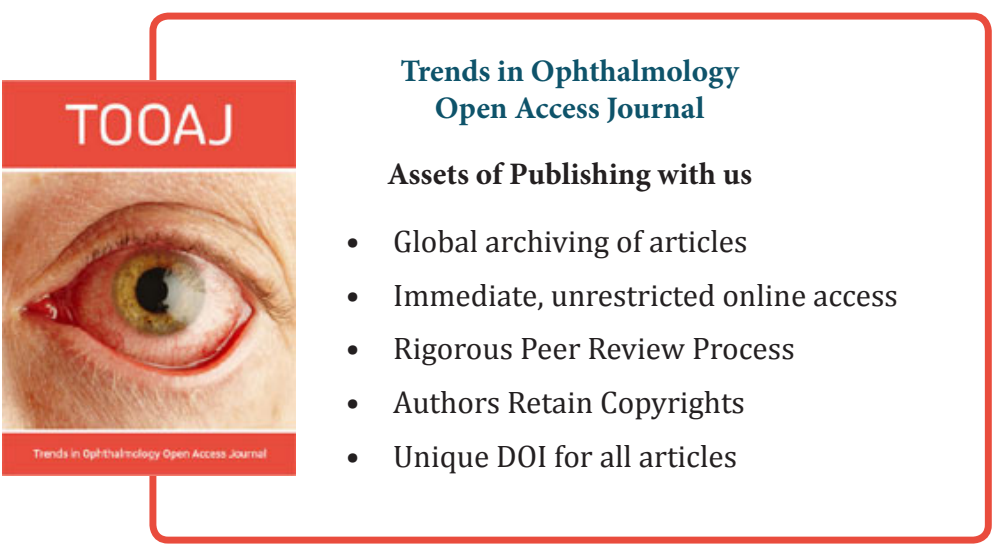

